\title{
Exercise and human gut microbiota: a systemic review
}

\section{Abstract}

Background: Eubiosis is the intestinal microbial ecosystem balance between human and microorganisms, whereas a disbalance in this intestinal microbial ecosystem is known as dysbiosis. The relationship between exercise with gut microbiota in humans is poorly studied, although it seems that one of the possible ways to restore eubiosis could be via exercise. This systematic review aimed to examine the scientific literature available on the influence of exercise in the gut microbiota of healthy adults.

Methods: A systematic and comprehensive literature search was conducted in PubMed and Web of Science (WOS) from their inception to April 2019. Search terms used were: "Gastrointestinal Microbiome", "Fecal Microbiota", "Cecal Microbiota", "Faecal Microbiota, "Exercises", "Training" and "Human".

Results: The initial search retrieved 218 articles and 15 met the inclusion criteria of which 9 were cross-sectional, 3 acute and 3 chronic exercise interventions. Higher levels of physical activity or $\mathrm{VO}_{2 \max }$ were positively associated with alpha-diversity in the $85.7 \%$ of the cross-sectional studies $(\mathrm{n}=6)$. We found controversial findings between levels of physical activity or $\mathrm{VO}_{2 \text { max }}$ with Firmicutes, Bacteroidetes and Proteobacteria phylum over cross-sectional studies. However, some studies found that higher levels of physical activity or $\mathrm{VO}_{2 \max }$ were positively related with Verrumicrobia and Actinobacteria, as well as their levels increased after the exercise interventions studies. Furthermore, higher levels of physical activity or $\mathrm{VO}_{2 \max }$ were positively related with short-chain-fatty-acids (SCFAs), as well as their levels increased after a chronic intervention.

Discussion: The muscle-gut axis is based on the contraction of skeletal muscle during exercise due to the release of myokines. This myokines that seem to play a role in mediating the glucagon-like peptide-1 (GLP-1) secretion in the gut during exercise. GLP-1 is one of the key incretins involved in the whole-body metabolism. On other hand, the gut-muscle axis, relies that the gut microbiota is able to produce SCFAs, which are mediator of mitochondrial energy metabolism in skeletal muscles.

Conclusion: Higher levels of physical activity or $\mathrm{VO}_{2 \max }$ are positively related with higher levels of alpha diversity and some phylum in healthy adults. Moreover, both acute and chronic exercises only influence some phylum. However, the high heterogeneity between studies hampers to draw stronger conclusions. Therefore, further studies are needed to understand the possible mechanism about how exercise could affect healthy human gut microbiota.

\section{Conflict of Interest}

There is no conflict of interest 